\title{
The Summary of Decision Making Problems in incomplete Soft Set
}

\author{
Lishi Zhang,LeiLeiQu and Ying Wan \\ School of Science, Dalian Ocean University, Dalian, China \\ dwtag@sohu.com
}

\begin{abstract}
Keywords: Soft Set; Choice Value; Weighted Average; incomplete fuzzy soft set; Relative Dominace Degree.
\end{abstract}

\begin{abstract}
In this paper, three approaches to deal with missing data in soft set are analyzed, Yan Zou[1] predicated the incomplete data with the average probability, the decision value of an object with incomplete information is calculated by weighted-average of all possible choice values of the objects. Zhi Kong[2]simplifies the approach and present the simplified probability to directly instead of the incomplete information. In paper[3],Deng introduces notions of complete distance between two objects and relative dominance degree between two parameters. An object-parameter method is proposed to predict unknown data in incomplete fuzzy soft set. In the current paper, a comparison is made to illustrate the advantages and disadvantages in all the approaches, a counterexample is given to show that in some cases the data provided by this approach is not guaranteed to be positive.
\end{abstract}

\section{Introduction}

The concept of soft set originated from the work of D. Molodtsov [4]. It is a parameterized family of subsets of a universe of discourse and is a powerful mathematical model in dealing with data sets with fuzziness and uncertainty. In a broad sense, a soft set can be represented by an information system or an information table intuitively. In the past, the theory of soft sets has been extensively explored[5,6,7,8]. Compared with other mathematical tools, such as probability theory, fuzzy set theory and rough set theory, a soft set model doesn't refer to prerequisite knowledge of the raw data sets. Soft sets can be generalized to some other structures such as with interval-valued fuzzy sets [9], interval-valued intuitionistic fuzzy soft set [10], the combination of soft sets with fuzzy sets [11], with rough set [12], with vague sets [13] still yield quite another structure.

Decision making in the field of a soft set is one of the most important tasks. An optimal choice for objects can be computed by summing the values of objects on all parameters or attributes. Once some entries of an information table are unknown, it is impossible to get any information to decision problems. In such case, the soft sets are called incomplete soft sets. If we delete all objects with missing entries, then the information is not complete. Zou and Xiao [1] presented a weighted-average method for soft sets. The missing values are predicted by weighted-average of all possible choice values of the object and the weight of each possible choice value is decided by the distribution of other objects. The weighted-average method can only predict the sum of values of every object instead of all parameters, each unknown entry in information tables cannot be estimated. The average-probability method can replace all unknown entry with the mean of the known data in one column, the predicted values of all unknown entries in one parameter column are all equal, this is not reasonable in reality. In this paper[3], Deng designs a novel method, called object-parameter method which predicts individual unknown entry in soft sets and in fuzzy soft sets. This method considers all information with objects and parameters, but in some situations, the data may not be positive.

The rest of this paper is arranged as follows. Section 2 recalls some fundamental concepts from soft set theory. Two classical methods of predicting unknown data for soft sets and fuzzy soft sets are reviewed in Section 3. A modified approach in response to Deng's method is given in Section 4. 


\section{Basic Concepts about soft sets}

This section recalls some basic notions on soft sets.

Definition 2.1. A pair $(F, E)$ is called a soft set (over $U$ ) if and only if $F$ is a mapping of $E$ into the set of all subsets of the set $U$, i.e., $F: E \rightarrow P(U)$, where $P(U)$ is the power set of $U$.

The soft set is a parameterized family of subsets of the set $U$. Every set $F(e), e \in E$, from this family may be considered as the set of $e$-elements of the soft set $(F, E)$, or as the $e$-approximate elements of the soft set.

Example 2.1. Let universe $U=\left\{h_{1}, h_{2}, h_{3}, h_{4}\right\}$ be a set of houses, a set of parameters $E=\left\{e_{1}, e_{2}, e_{3}\right.$, $e_{4}$ \} be a set of status of houses which stand for the parameters "beautiful", "cheap", "in green surroundings", and "in good location" respectively. The mapping $F$ be a mapping of $E$ into the set of all subsets of the set $U$. Now consider a soft set $(F, E)$ that describes the "attractiveness of houses for purchase", where

$$
F\left(e_{1}\right)=\left\{h_{1}, h_{3}, h_{4}\right\}, F\left(e_{2}\right)=\left\{h_{1}, h_{2}\right\}, F\left(e_{3}\right)=\left\{h_{1}, h_{3}\right\} \text {, and } F\left(e_{4}\right)=\left\{h_{2}, h_{3}, h_{4}\right\} .
$$

A two-dimensional table is used to represent the soft set $\{F, E\}$. Table $\mathrm{I}$ is the tabular form of the soft set $\{F, E\}$. If $h_{\mathrm{i}} \in F\left(e_{\mathrm{j}}\right)$, then $h_{\mathrm{ij}}=1$, otherwise $h_{\mathrm{ij}}=0$, where $h_{\mathrm{ij}}$ are the entries (see Table I). Suppose

$$
U=\left\{h_{1}, h_{2}, \ldots \ldots, h_{\mathrm{n}}\right\}, E=\left\{e_{1}, e_{2}, \ldots \ldots, e_{\mathrm{m}}\right\},
$$

$(F, E)$ is a soft set with tabular representation. Define, $f_{E}\left(h_{i}\right)=\sum_{j}\left\{h_{i j}\right\}$, where $h_{\mathrm{ij}}$ are the entries in soft set table.

TABLE I. THE TABULAR REPRESENTATION OF $\{H, A\}$

\begin{tabular}{|l|l|l|l|l|l|l|l|l|l|l|}
\hline$U$ & $e_{1}$ & $e_{2}$ & $e_{3}$ & $e_{4}$ & $e_{5}$ & $e_{6}$ & $e_{7}$ & $e_{8}$ & $e_{9}$ & $e_{10}$ \\
\hline$h_{1}$ & 1 & 0 & 1 & 0 & 1 & 1 & 1 & $*$ & 1 & 0 \\
\hline$h_{2}$ & 0 & 1 & 1 & $*$ & 0 & 1 & 0 & 1 & 1 & 1 \\
\hline$h_{3}$ & 1 & 1 & 0 & 1 & 1 & 1 & 1 & 0 & 1 & 1 \\
\hline$h_{4}$ & 1 & $*$ & 0 & 1 & 0 & 0 & 0 & 0 & 1 & 1 \\
\hline$h_{5}$ & 1 & 0 & 1 & 0 & 1 & 1 & $*$ & 0 & $*$ & 0 \\
\hline$h_{6}$ & 1 & 0 & 1 & $*$ & 0 & 1 & 1 & $*$ & 1 & 1 \\
\hline$h_{7}$ & 1 & 1 & 0 & 1 & 1 & 1 & 0 & 0 & 1 & 0 \\
\hline$h_{8}$ & $*$ & 1 & 1 & 1 & 0 & 0 & 0 & 1 & 1 & 0 \\
\hline$h_{9}$ & 0 & 0 & 0 & 1 & 1 & 0 & 1 & 1 & 0 & 0 \\
\hline$h_{10}$ & 0 & 1 & 0 & 1 & 1 & 1 & $*$ & 0 & 1 & 1 \\
\hline$h_{11}$ & 1 & 0 & 1 & 1 & 1 & 1 & 0 & 0 & $*$ & 1 \\
\hline$h_{12}$ & 0 & 0 & 0 & 1 & 1 & 0 & 0 & 0 & 1 & 0 \\
\hline
\end{tabular}

The following table shows the two approaches of average-probability and weighted-average respectively.

TABLE II. THE TABULAR REPRESENTATION OF $\{H, A\}$

\begin{tabular}{|l|l|l|}
\hline$U$ & average-probability & weighted-average \\
\hline$h_{1}$ & 6.5 & 6.3 \\
\hline$h_{2}$ & 6.5 & 6.5 \\
\hline$h_{3}$ & 7 & 7 \\
\hline$h_{4}$ & 5.5 & 5.45 \\
\hline$h_{5}$ & 6 & 6.3 \\
\hline$h_{6}$ & 7 & 6.8 \\
\hline$h_{7}$ & 6 & 6 \\
\hline$h_{8}$ & 3.5 & 3.64 \\
\hline$h_{9}$ & 5 & 5 \\
\hline$h_{10}$ & 7.5 & 7.4 \\
\hline$h_{11}$ & 6.5 & 6.9 \\
\hline$h_{12}$ & 2 & 2 \\
\hline
\end{tabular}




\section{Proposed Approaches}

In Deng's approach, let $(F, E)$ be a fuzzy soft set over $U$, for $h_{\mathrm{i}}, h_{\mathrm{j}} \in U$ and $\mathrm{e}_{\mathrm{k}} \in \mathrm{E}$, the relative distance from $h_{\mathrm{i}}$ to $h_{\mathrm{j}}$ with respect to $\mathrm{e}_{\mathrm{k}}$ is denoted by

$$
d_{i j, k}=\frac{h_{i k}-h_{j k}}{\sum_{l \in U_{k}}\left|h_{l k}-h_{j k}\right|}
$$

$d_{i j}$ is the complete distance between the values of objects $h_{\mathrm{i}}$ and $h_{j}$ on all the parameters. The complete distance $d_{i j}$ can be positive or negative, it is defined as

$$
d_{i j}=\frac{\sum_{k=1}^{n} d_{i j, k}}{\left|\left\{k \mid\left(i \in U_{k}\right) \wedge\left(j \in U_{k}\right)\right\}\right|}
$$

the unknown entry $h_{\mathrm{j}}$ is evaluated according to the information from the relationship between the values of objects on a certain parameter by

$$
h_{j l}^{\text {object }}=\frac{\sum_{i \in U_{l}}\left(h_{i l}-d_{i j}\right)}{\left|U_{l}\right|}
$$

But in some cases, $h_{j l}^{\text {object }}$ may be negative.

TABLE III. THE TABULAR REPRESENTATION OF $\{H, A\}$
\begin{tabular}{|l|l|l|l|}
\hline$U$ & $e_{1}$ & $e_{2}$ & $e_{3}$ \\
\hline$h_{1}$ & 0.3 & 0.2 & 0.1 \\
\hline$h_{2}$ & 0.2 & 0.1 & $*$ \\
\hline$h_{3}$ & 0.3 & 0.2 & 0.2 \\
\hline
\end{tabular}

table IV. The TABUlar REPRESENTATION of $\{H, A\}$

\begin{tabular}{|l|l|l|l|}
\hline$U$ & $e_{1}$ & $e_{2}$ & $e_{3}$ \\
\hline$h_{1}$ & 0.3 & 0.2 & 0.1 \\
\hline$h_{2}$ & 0.2 & 0.1 & $-0.35(0.35)$ \\
\hline$h_{3}$ & 0.3 & 0.2 & 0.2 \\
\hline
\end{tabular}

$$
\begin{aligned}
& d_{12,1}=\frac{h_{11}-h_{21}}{\left|h_{11}-h_{21}\right|+\left|h_{31}-h_{21}\right|}=\frac{0.3-0.2}{|0.3-0.2|+|0.2-0.1|}=0.5 \\
& d_{12,2}=\frac{h_{12}-h_{22}}{\left|h_{12}-h_{22}\right|+\left|h_{32}-h_{22}\right|}=\frac{0.2-0.1}{|0.2-0.1|+|0.2-0.1|}=0.5 \\
& d_{32,1}=\frac{h_{31}-h_{21}}{\left|h_{31}-h_{21}\right|+\left|h_{11}-h_{21}\right|}=\frac{0.3-0.2}{|0.3-0.2|+|0.3-0.2|}=0.5 \\
& d_{32,2}=\frac{h_{32}-h_{22}}{\left|h_{32}-h_{22}\right|+\left|h_{12}-h_{22}\right|}=\frac{0.2-0.1}{|0.2-0.1|+|0.2-0.1|}=0.5 \\
& d_{12}=\frac{1}{2}\left(d_{12,1}+d_{12,2}\right)=0.5 ; d_{32}=\frac{1}{2}\left(d_{32,1}+d_{32,2}\right)=0.5 \\
& h_{23}^{\text {object }}=\frac{\sum_{i \in U_{3}}\left(h_{i 3}-d_{i 2}\right)}{\left|U_{3}\right|}=\frac{\left(h_{13}-d_{12}\right)+\left(h_{33}-d_{32}\right)}{\left|U_{3}\right|}=-0.35
\end{aligned}
$$

As $h_{23}^{\text {object }}<0$, therefore, the algorithm should be altered, it is clear that $\left|d_{i j, k}\right| \leq 1$, and $\left|d_{i j}\right| \leq 1$, so it is sure that $\left|h_{j l}^{\text {object }}\right| \leq 1$, but it is not necessarily to be positive. So we can replace the data by its abstract value instead.

The relationship between values of an object on all parameters is also very important in predicting unknown data in an incomplete fuzzy soft set. In Deng's approach, let $(F, E)$ be a fuzzy soft set over $U$, for $h_{i} \in U$ and $e_{k}, e_{l} \in E$, the degree of $e_{k}$ being relatively dominant to $e_{l}$ regarding $h_{i}$ is defined by

$$
r_{i, k l}=\frac{h_{i k}-h_{i l}}{h_{i k}+h_{i l}}
$$


The degree of $e_{k}$ being definitely dominant to $e_{l}$ is defined by

$$
c_{k l}=\frac{\sum_{i \in U_{k} \cap U_{l}} r_{i, k l}}{\left|U_{k} \cap U_{l}\right|}
$$

the degree of average dominance of $e_{k}$ to $e_{l}$ is characterized by

$$
v_{k l}=\frac{c_{k l}}{\sum_{\left\{q \mid U_{q} \cap U_{l} \neq \varnothing\right\}}\left|c_{q l}\right|}
$$

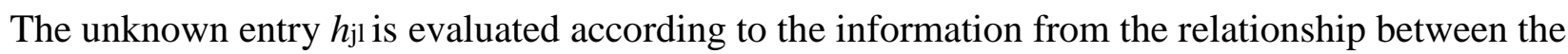
parameters regarding objects $h_{j}$ by

From the above table,

$$
h_{j l}^{\text {parameter }}=\frac{\sum_{k \in E_{j}}\left(h_{j k}-v_{k l}\right)}{\left|E_{j}\right|}
$$

$$
\begin{aligned}
& r_{1,23}=\frac{h_{12}-h_{13}}{h_{12}+h_{13}}=1 / 3 ; r_{3,23}=\frac{h_{32}-h_{33}}{h_{32}+h_{33}}=0 ; r_{1,13}=\frac{h_{11}-h_{13}}{h_{11}+h_{13}}=0.5 ; r_{3,13}=\frac{h_{31}-h_{33}}{h_{31}+h_{33}}=0.2 ; \\
& c_{23}=\frac{1}{2}\left(r_{1,23}+r_{3,23}\right)=1 / 6 ; c_{13}=\frac{1}{2}\left(r_{1,13}+r_{3,13}\right)=0.35 ; v_{13}=\frac{c_{13}}{\left|c_{23}\right|+\left|c_{13}\right|}=0.6774 ; v_{23}=\frac{c_{23}}{\left|c_{13}\right|+\left|c_{23}\right|}=0.3226 ; \\
& h_{23}^{\text {parameter }}=\frac{\sum_{k \in E_{j}}\left(h_{2 k}-v_{k 3}\right)}{\left|E_{j}\right|}=\frac{\left(h_{21}-v_{13}\right)+\left(h_{22}-v_{23}\right)}{\left|E_{j}\right|}=-0.35
\end{aligned}
$$

As $h_{23}^{\text {parameter }}<0$, therefore, the algorithm should be changed, as $\left|r_{i j, k}\right| \leq 1$, and $\left|v_{i j}\right| \leq 1$, so it is sure that $\left|h_{j l}^{\text {parameter }}\right| \leq 1$, but it is not necessarily to be positive. In both cases, we can replace the data with its abstract value, that is

$$
h_{j l}^{\text {object }}=\frac{\left|\sum_{i \in U_{l}}\left(h_{i l}-d_{i j}\right)\right|}{\left|U_{l}\right|} ; h_{j l}^{\text {parameter }}=\frac{\left|\sum_{k \in E_{j}}\left(h_{j k}-v_{k l}\right)\right|}{\left|E_{j}\right|}
$$

\section{References}

[1]Y.Zhou, Z.Xiao, Data analysis approaches of soft sets under incomplete information, Knowledge-Based Systems 21 (2008) 941-945

[2]Z.Kong, G.D.Zhang,L.F.Wang, An efficient decision making approach in incomplete soft set , Applied Mathematical Modelling 38(2014) 2141-2150.

[3]T.Q.Deng, X.F.Wang, An object-parameter approach to predicting unknown data in incomplete fuzzy soft sets, Applied Mathematical Modelling 37(2013)4139-4146.

[4] D. Molodtsov. “Soft set theory-first results," Comput. Math. Appl, Vol 37, 1999, pp.19-31.

[5] Y.B. Jun, K.J. Lee, C.H. Park, Fuzzy soft set theory applied to BCK/BCI-algebras, Comput. Math. Appl. 59 (2010) 3180-3192.

[6] P.K. Maji, R. Biswas, A.R. Roy, Fuzzy soft sets, J. Fuzzy Math. 9 (3) (2001) 589-602.

[7] F. Feng, Xiaoyan Liu, Violeta Leoreanu-Fotea, Y.B. Jun, Soft sets and soft rough sets, Inform. Sci. 181 (2011) 1125-1137.

[8]Y. Jiang, H. Liu, Y. Tang, Q. Chen, Semantic decision making using ontology-based soft sets, Math. Comput. Model. 53 (2011) 1140-1149.

[9] D. Chen, E.C.C. Tsang, Daniel S. Yeung, X. Wang, The parameterization reduction of soft sets and its applications, Comput. Math. Appl. 49 (2005) 757-763. 
[10]J. Zhan, Y.B. Jun, Soft BL-algebras based on fuzzy sets, Comput. Math. Appl. 59 (2010) 2037-2046.

[11] Wei Xu, Jian Ma, Shouyang Wang, Gang Hao, Vague soft sets and their properties, Comput. Math. Appl. 59 (2010) 787-794. 